\title{
AC Electrical Conductivity of Cr-Doped Polyaniline/Poly(Vinyl alcohol) Blends
}

\author{
D.A.W. Soares*, P. H. O. de Souza*, R. M. Rubinger*, A.A.A. de Queiroz*, \\ O.Z. Higa ${ }^{\dagger}$, and L.R. de Souza* \\ * Departamento de Física e Química, Instituto de Ciências, Universidade Federal de Itajubá \\ Campus José Rodrigues Seabra, Av. BPS, 1303, 37500-903, Itajubá, MG, Brazil \\ ${ }^{\dagger}$ Laboratório de Biologia Molecular, Instituto de Pesquisas Energéticas e Nucleares, IPEN-USP, SP, Brazil
}

Received on 31 March, 2003

\begin{abstract}
Polyaniline (PANI) and their charge transfer complexes are engineering materials that continue to attract considerable attention from engineers due to their unique electrochemical behavior, environmental stability and high degree of processability. Extensive works on the preparation of electrically conductive PANI blends containing a polymeric matrix having desirable physical and good mechanical characteristics have pointed as a promising route to overcome the PANI intractabilities. This paper reports the electrical properties of the dichromated PANI/poly(vinyl alcohol) (PANI:Cr $\left.{ }^{3+} / \mathrm{PVA}\right)$ blends. Doped polyaniline powder in the emeraldine base form was synthesized by the classical chemical oxidation of aniline with ammonium persulfate as the oxidant in the presence of chromium $\left(\mathrm{Cr}^{3+}\right)$. The SEM (Phillips XL 30) was used to understand the changes occurring in the conductivity to a microstructure level. The distribution of PANI: $\mathrm{Cr}^{3+}(50 \% \mathrm{w} / \mathrm{w})$ in the host polymer (PVA) show some tendency to occur in "flocculated structures". The samples were cut into circular shapes to form films about 500 microns thick. Electrical contacts were made by sputtering gold over sample surfaces. We have carried out impedance spectroscopy measurements in the range of $20 \mathrm{~Hz}$ to $1 \mathrm{MHz}$. Measurements carried at $100 \mathrm{~K}$ and $250 \mathrm{~K}$ indicates that thermally activated carrier process between PANI: $\mathrm{Cr}^{3+}$ grains in the PANI:Cr ${ }^{3+} / \mathrm{PVA}$ blend sample are responsible for its high temperature conductivity.
\end{abstract}

\section{Introduction}

The intrinsically conductive polymers have been attracting the attention of research countless groups since their discovery, mainly by the scientific importance in new phenomena and by their potential technological applications. Such polymers can combine the mechanical characteristics and ease processing of the conventional ones with electrical, optical and also magnetical properties, which sometimes resemble metals and inorganic semiconductors. These characteristics turn conductive polymers into the so-called synthetic metals category [1].

Therefore, polyaniline and aniline derivatives have received great attention recently [2] considering their chemical stability in environmental conditions, simple and cheap polymerisation process and doping. These advantages can be applied to several technological and industrial applications [3].

\section{Materials and Methods}

Polyaniline (PANI) was chemically synthesized as emeraldine salt, in part, by oxidative polymerization of aniline in $1 \mathrm{M}$ aqueous $\mathrm{HCl}$ solution with ammonium persulphate as described in the literature [4]. The first step consisted in aniline distillation at $170^{\circ} \mathrm{C}$. Ammonium persulphate was the oxidizer agent which turns in the polyaniline form. Than polyaniline, in its emeraldine base form, was doped using $\mathrm{HCl}$ dissolved in $\mathrm{N}$-methyl (2-pyrrolidone) in the chromium presence (i.e. $20 \%$ of $\mathrm{Cr}^{+3}$ in the solution). The obtained chromium doped polyaniline (PANI: $\mathrm{Cr}^{+3}$ ) was then washed with propyl alchohol in order to remove all low-weight molecular materials. Finally, poly (vinyl alcohol) (PVA) was added to this solution. Thus, the obtained material is a polidisperse film with dark green color, denominated emeraldine salt ( $50 \%$ w/w) - (PANI:Cr $\left.{ }^{+3} / \mathrm{PVA}\right)$.

The electrical properties of the PANI:Cr ${ }^{+3} / \mathrm{PVA}$ were evaluated by impedance spectroscopy measurements [5]. Through electrical measurements, the dielectric constant, dielectric loss and other material properties could be obtained.

For electrical measurements, the samples were cut into thin disk shapes and sputtered on two opposite sides with gold. The impedance analyzer HP4192A was used in this work to evaluate dielectrical relaxation phenomena.

\section{Results and Discussion}

The microstructure of the blends, characterized by scanning electron microscopy (SEM) is presented in Fig. 1. In this Figure it is shown the PANI: $\mathrm{Cr}^{+3}$ grains embedded in the PVA matrix.

Under ac electric field, the dielectric properties change with frequency. Fig. 2 and Fig. 3 presents impedance $(Z)$ 


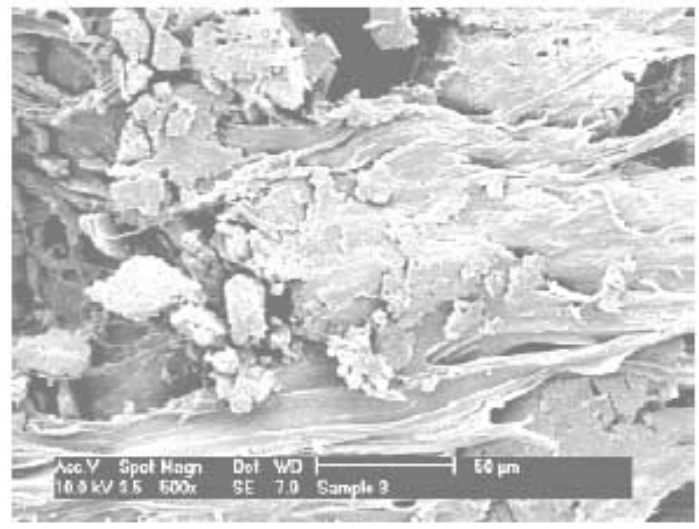

Figure 1. Scanning electron micrographs of the fracture of PANI:Cr ${ }^{+3} / \mathrm{PVA}$.

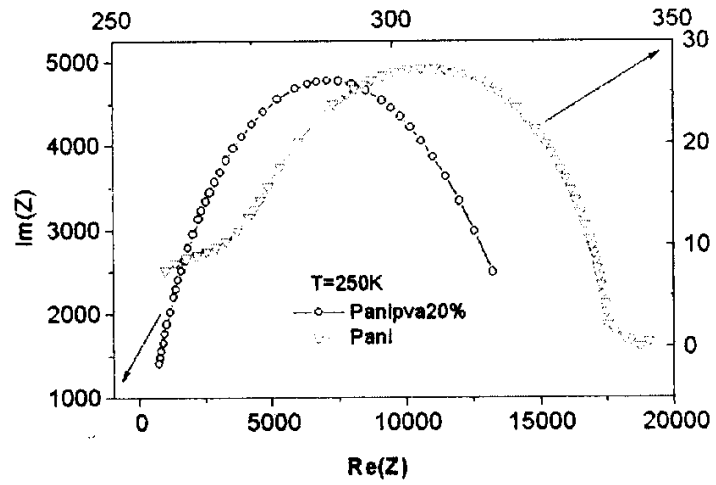

Figure 2. $\mathrm{Z}$ arcs for undoped PANI in open circles and down triangles for PANI: $\mathrm{Cr}^{3+} / \mathrm{PVA}$ at $250 \mathrm{~K}$. The corresponding axes are indicated.

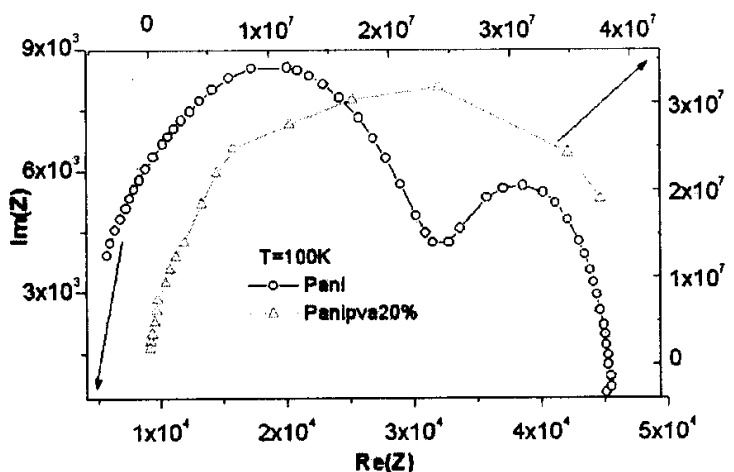

Figure 3. $\mathrm{Z}$ arcs for undoped PANI in open circles and up triangles for PANI:Cr ${ }^{3+} / \mathrm{PVA}$ measured at $100 \mathrm{~K}$.

arc for the PANI:Cr ${ }^{3+} / \mathrm{PVA}$ and for a undoped PANI sam- ples measured at $250 \mathrm{~K}$ and $100 \mathrm{~K}$ respectively. In Fig. 2 we see that the undoped PANI represented by open circles presenting lower impedance than the PANI: $\mathrm{Cr}^{3+} / \mathrm{PVA}$ sample shown by open triangles indicating that, although the PVA is a good insulator, its combination with PANI: $\mathrm{Cr}^{3+}$ results in conductive samples. At this temperature the blended sample is one magnitude order less conductive than the undoped PANI. This fact will be very interesting when compared with the data on Fig. 3.

Fig. 3 presents measurements carried at $100 \mathrm{~K}$. At this temperature the undoped PANI sample has become 100 times conductive but the PANI: $\mathrm{Cr}^{3+} / \mathrm{PVA}$ sample has become much more insulator. Open circles represent the measurements for the undoped PANI and open triangles the measurements for PANI:Cr ${ }^{3+} / \mathrm{PVA}$. At this temperature the blended sample is much more insulator than the undoped PANI. Also PANI characteristics present two Z arcs which might be due to contact effects. The difference observed in the conductance of the blended sample might be associated with a thermally activated process between PANI:Cr ${ }^{3+}$ grains. In a temperature between $100 \mathrm{~K}$ and $250 \mathrm{~K}$ the PANI:Cr ${ }^{3+} / \mathrm{PVA}$ sample changes its conductive behavior into insulator one. Further studies will bring these changes into evidence. Thermally activated carriers and thermal vibrations of the sample structure might give rise to carrier tunneling between PANI:Cr ${ }^{3+}$ grains. Thermal vibrations are known to facilitate hopping conduction in lightly doped semiconductors [6].

Our results are still not enough to any conclusive indication regarding hopping conduction. We intend to carry, in a near future, detailed resistivity studies in order to identify the variable range hopping $\mathrm{T}^{-1 / 4}$ signature. Due to sample capacitance, d.c. measurements are very slow and in a.c. measurements there is a combination of the imaginary part of the frequency dependent electrical permittivity with the contribution of the resistivity. For this we will need to separate both contributions.

From the impedance arc we can estimate the relaxation time $\tau_{0}$ assessed by the inverse of the angular frequency at the maximal loss, i.e. $\tau_{0}=1 / \omega \max$ [7].

The relaxation times obtained for the data presented in Figs. 2 and 3 are listed in Table 1. At 250K there is a difference of one order of magnitude between both samples and at $100 \mathrm{~K}$ the difference is much less significant. These data confirms the changes observed in the sample conductive behavior, since more conductive samples present lower relaxation times.

Table 1. Relaxation times for the $\mathrm{Z}$ arcs shown in Figs. 2 and 3.

\begin{tabular}{|l|l|l|}
\hline Sample & $\begin{array}{l}\text { Relaxation time } \\
\text { for } \mathrm{T}=100 \mathrm{~K}(\mathrm{~s})\end{array}$ & $\begin{array}{l}\text { Relaxation time } \\
\text { for } \mathrm{T}=250 \mathrm{~K}(\mathrm{~s})\end{array}$ \\
\hline undoped PANI & $2.67 \times 10^{-4}$ & $3.18 \times 10^{-5}$ \\
\hline PANI:Cr ${ }^{3+} / \mathrm{PVA}$ & $2.26 \times 10^{-4}$ & $3.83 \times 10^{-6}$ \\
\hline
\end{tabular}




\section{Summary}

We have carried out frequency response analysis on undoped polyaniline and a blend of dichromated polyaniline with poly(vinyl alcohol) at two low temperatures, i.e. $100 \mathrm{~K}$ and $250 \mathrm{~K}$. The results indicates that at the highest temperature the blended sample is more conductive while at the lowest temperature the undoped polyaniline is the most conductive one. The change in the characteristics of the blended sample between the two temperatures is radical and might be associated with a conductive process thermally activated. Relaxation times for both samples obtained at $100 \mathrm{~K}$ and $250 \mathrm{~K}$ follows the changes in sample resistivity.

\section{Acknowledgements}

We would like to acknowledge the Brazilian agencies CNPq, CAPES and FAPEMIG for financial support. [FAPEMIG CEX 1520/98, Capes]

\section{References}

[1] V.E. Bondarenko, T.S. Zhuravleva, O.N. Efimov, and G.V. Nikolaeva, Syn. Met. 102, 1228 (1999).

[2] D.A.W. Soares, A.A.A. Queiroz, Macromolecular Symposia, 170, 221 (2001).

[3] L.H.C. Mattoso, Química Nova, 19, 388 (1996).

[4] Suzhu Yu, Peter Hing, and Xiao Hu, J. App. Phys. 88, 398 (2000).

[5] P. Dutta, S. Biswas, and Subodh Kumar De, Mater. Res. Bull. 37, 193 (2002).

[6] A.L. Efros, N. V. Lien, and B.I. Shklovskii, Solid Stat. Comm. 32, 851 (1979).

[7] N.G. McCrum, B.E. Read, and G. Williams in: Anelastic and Dielectric Effects in Polymeric Solids, Dover Publications, Inc. New York. (1991). 